\title{
TPACK: Aplicabilidad docente del modelo en Educación General Básica Elemental
}

\section{TPACK: Teaching applicability of the model in Basic Elementary General Education}

TORRES, Carlos A. ${ }^{1}$

ESPINOSA, Willan A. ${ }^{2}$

ROMERO, Daniela M. ${ }^{3}$

HERRERA, Rosario $\mathrm{S}^{4}$

HERRERA, Diego A. ${ }^{5}$

\begin{abstract}
Resumen
Este estudio analiza la aplicabilidad de relacionar el modelo del Conocimiento Tecnológico Pedagógico del Contenido (TPACK) con la práctica docente apoyada por las Tic en Educación General Básica Elemental, a través de un estudio científico descriptivo-correlacional, que parte de la caracterización de ambos enfoques y se centra en la valoración de sus implicaciones didácticas desde una óptica integral. Como conclusión, la propuesta constituye un marco de integración positivo, idóneo y eficaz de la tecnología para la educación.

Palabras clave: aplicabilidad docente, modelo tpack, educación general básica elemental

Abstract

This study analyzes the applicability of relating the Pedagogical Technological Knowledge of Content (TPACK) model with the teaching practice supported by ICT in Basic Elementary General Education, through a descriptive-correlational scientific study, which starts from the characterization of both approaches, and it focuses on the evaluation of its didactic implications from an integral perspective. In conclusion, the proposal constitutes a positive, suitable and effective integration framework for technology for education.
\end{abstract}

Key words: teaching applicability, tpack model, basic elementary general education

\section{Introducción}

La idea de vincular las Tic con el proceso de aprendizaje y la necesidad de que el docente desarrolle, adquiera y recicle nuevas competencias, están relacionadas, al ámbito de la formación y al empleo educativo de las tecnologías, donde, según Cabero, Marín y Castaño, citados por Barajas y Cuevas (2017) se han encontrado dos problemas fundamentales: primero, mencionan la excesiva tecnificación en torno a los cursos que se diseñan y llevan a cabo y segundo, señalan la falta de modelos conceptuales didácticos para dirigir la capacitación del

\footnotetext{
${ }^{1}$ Licenciado en Informática Educativa. Carrera de Informática Educativa. Universidad Nacional de Loja. carlos.a.torres@unl.edu.ec.

${ }^{2}$ Doctor en psicología educativa y orientación, Magíster en desarrollo educativo. Carrera de psicología. Universidad Técnica Particular de Loja. waespinosa@utpl.edu.ec.

${ }^{3}$ Magister en Pedagogía. Carrera de Psicología Infantil y Educación Parvularia. Universidad Nacional de Loja. maria.d.suarez@unl.edu.ec.

${ }^{4}$ Magister en Educación Especial. Licenciatura en Ciencias de la Educación. Escuela Miguel Riofrio. herrera_charito@hotmail.com.

${ }^{5}$ Licenciado en Ciencias de la Educación mención: Físico Matemáticas. Unidad Educativa Fiscomisional "La Dolorosa". dihego_0286@hotmail.com.
} 
docente la cual se supone, deberá proveer los conocimientos, habilidades, recursos y destrezas necesarias para integrar de manera eficaz éstas a la práctica profesional.

Los primeros estudios del modelo TPACK se lo atribuye a Shulman, (1986) fueron Mishra y Koehler (2009) quienes añadieron a esa teoría otro tipo de conocimiento, el conocimiento tecnológico, que integra lo pedagógico y el contenido. Con la expansión de la tecnología, Dussel citado por Castillejos, Torres, Lagunes, (2016), reflexiona entorno a los desafíos pedagógicos que implica introducir nuevas tecnologías en las escuelas, tanto en términos de la reestructuración del espacio y del tiempo, como en la reorganización de los saberes y las relaciones de autoridad.

Referente a esto, se espera que las Tic generen un cambio en el sistema educativo, ya que siempre han sido utilizadas como instrumentos para aprender, conocer y transmitir a otras personas los conocimientos $y$ aprendizajes adquiridos, las mismas que ha contribuido a transformar y replantear los escenarios y las prácticas educativas mediadas por las Tic (Coll, 2004). En la actualidad, los docentes tienen la oportunidad de incorporar la tecnología para organizar las actividades escolares motivando su uso dentro y fuera del salón de clase; es dentro de este esquema que se ubica la presente investigación en la que se analiza mediante un estudio científico descriptivo-correlacional, la aplicabilidad del modelo TPACK en la práctica docente de las instituciones educativas fiscales, fiscomisionales y municipales del Distrito 11D01 en la ciudad de Loja.

\subsection{El modelo TPACK}

Este término originalmente fue presentado por Lee Shulman (1986) como conocimiento pedagógico disciplinar o PCK (Pedagogical Content Knowledge), a partir del cual se desarrolla un nuevo término, la concepción del TPACK (Technological Pedagogical Content Knowledge) este modelo fue planteado por Punya Mishra y Koehler, en una serie de publicaciones entre el 2006 y 2009. (Gomez, 2018); el modelo hace referencia a la relación entre el conocimiento tecnológico, pedagógico y disciplinar.

Para Shulman el conocimiento del contenido y el conocimiento pedagógico son más que dos saberes paralelos para el docente, son en realidad complementarios porque hacen intersección y dan paso a un tercero el conocimiento pedagógico del contenido; ahora con la proliferación de internet y la web 2.0 surge un nuevo conocimiento el tecnológico, autores como Mishra y Koehler citados por Vallejo (2013) muestra, que este saber incluye nuevas intersecciones que dan paso al modelo TPACK el cual consta de los conocimientos que todo docente debe tener para incorporar las Tic en los escenarios formativos. Para ello, es necesario partir de una serie de premisas a la hora de afrontar un modelo de integración en las clases; existen 7 zonas distintas de conocimientos (componentes) integrados a partir de los saberes pedagógicos, disciplinares y tecnológicos, en donde se manifiesta que todos estos conocimientos deben ser contemplados de forma individual y en su mutua interacción:

1. Conocimientos sobre el contenido de la materia (CK), hace referencia a lo que se enseña y aprende como teorías, conceptos, procesos y procedimientos de un campo determinado.

2. Conocimiento pedagógico (PK), se refiere a los procesos y prácticas, métodos de enseñanza-aprendizaje, valores y objetivos en general, con fines educativos, así como la construcción del conocimiento y desarrollo de competencias por parte de los estudiantes.

3. Conocimiento tecnológico (TK), se trata de los conocimientos y las competencias que los docentes deben tener respecto a cómo las Tic pueden apoyar su actividad profesional de enseñanza y cómo estas contribuyen al aprendizaje de los estudiantes. 
Al relacionar estos tres conocimientos surgen otros, estos son:

4. Conocimiento pedagógico del contenido (PCK), hace referencia a un área concreta y por tanto es diferente para otra; incluye la comprensión de las representaciones sobre temas específicos en una disciplina determinada y el conocimiento de la pedagogía y la didáctica propia de cada saber, de dónde se deriva por ejemplo la enseñanza de las ciencias, las artes, la lengua y de las ciencias sociales.

5. Conocimiento tecnológico pedagógico (TPK), hace referencia a cómo las Tic pueden usarse en la enseñanza y el modo en que su uso puede cambiar la forma de educar y organizar el ambiente de trabajo en el aula, involucrando a los estudiantes en el aprendizaje colaborativo.

6. Conocimiento Tecnológico del contenido (TCK), este conocimiento alude a cómo hacer representaciones de conceptos con la tecnología; así como al software especializado para cada área.

Finalmente, si relacionamos los seis conocimientos básicos e extrae el conocimiento con experiencia del docente en materia Tic, el TPACK.

7. Conocimiento tecnológico, pedagógico del contenido (TPACK), el cual se refiere a la utilización de las Tic para la representación de la información y el desarrollo de actividades, así como la interacción y participación para facilitar el aprendizaje del estudiante.

El modelo ofrece un marco de entendimiento para mejorar los conocimientos pedagógicos, tecnológicos y disciplinares de los docentes para que las propuestas educativas se centren en el estudiante, el currículo y propicien una integración metodológica de lo tecnológico adecuada a los nuevos escenarios de aprendizaje. Sin embargo, por complejo que puede hacer este conocimiento lo es aún más, debido a los múltiples contextos en el uso de Tic como la disponibilidad de tecnologías, tiempo, espacio, las diferencias culturales económicaseducativas entre otras en el que los estudiantes y docentes trabajan juntos.

\subsection{La aplicación del modelo TPACK}

Autores como González (2017) han realizado estudios sobre cómo influye el contexto en el desarrollo del conocimiento, ya que todo aprendizaje es situado y por tanto no es posible generalizar ninguna práctica, por ende una actividad no funciona en todos los contextos; además es importante entender que el conocimiento es comunicativo y transversal porque se entrelazan la comunicación de un saber y sus diversas formas de presentación como lo comunicativo dentro de lo pedagógico y didáctico lo cual es fundamental, pues no se concibe la educación sin comunicación.

Algunos estudios empíricos sobre TPACK revelan que los objetivos pedagógicos de los docentes definen el tipo de tecnología a emplear. Este modelo es un objetivo en movimiento, donde cada docente tiene su base de conocimiento y su aplicación varía de acuerdo al contexto donde se desarrolle el proyecto educativo. Por otro lado, se ha detectado que los docentes que no posean competencias sobre pedagogías flexibles y de aprendizaje auténtico, experimentarán mayores dificultades en su aplicación ya que la falta de estas capacidades frena el desarrollo de propuestas tecno-educativas. (Castillejos, Torres Gastelú, y Lagunes Dominguez, 2016, p. 51)

Harris, J. y Hofer, M. citados por Castillejos, Torres y Lagunes (2016), proponen una planeación didáctica basada en las actividades educativas. Tras años de investigación han desarrollado estudios en diversas áreas de interés como: alfabetización en preescolar y primaria, matemáticas, música, educación física, ciencias, inglés como segunda lengua, ciencias sociales, artes visuales y lenguas extranjeras. El producto de tales investigaciones se ven reflejadas en tablas taxonómicas que presentan el tipo de actividad acompañada de una breve descripción y las tecnologías sugeridas a emplear. En este sentido, los autores resaltan tres beneficios del modelo: primero 
la selección y uso de actividades y tecnologías de aprendizaje se realizan a conciencia; segundo la planificación de la instrucción más centrada en el estudiante; y tercero se definen normas para la integración de la tecnología.

De acuerdo con Adell (2012) la aplicación en el aula parte de una toma de decisiones, iniciando por elegir qué contenido se quiere trabajar, tomando en cuenta los objetivos del área, así como revisar las competencias requeridas y criterios de evaluación. Posterior a ello, se toman otras decisiones sobre los recursos materiales y la naturaleza de la actividad didáctica, después se seleccionan y organizan los tipos de actividades a combinar para crear una secuencia didáctica y por último, se opta por las estrategias de evaluación de la experiencia (Harris, 2012). Es importante comentar que todo dependerá del contexto donde se desarrolle la práctica, así como el grado de participación de las autoridades educativas para apoyar la gestión del TPACK.

\subsection{El modelo TPACK y las Tic}

Al utilizar el modelo TPACK para enseñar con Tic de una forma eficaz es necesario que el docente domine los tres componentes básicos: contenido, pedagogía y tecnología. Sin embargo Gomez (2018) manifiesta que tras un detallado análisis de los principios del modelo se pueden también tomar en consideración los siguientes aspectos:

- Modelo relacional. El dominio del contenido, la pedagogía y la tecnología no aseguran por sí solos una enseñanza eficaz integrando Tic; se trata no sólo de dominar el contenido y las estrategias de enseñanza/aprendizaje sino también saber qué herramientas tecnológicas utilizar y cómo se pueden aplicar teniendo en cuenta que a su vez su uso pueden modificar los contenidos y las propias dinámicas de enseñanza y aprendizaje.

- Toma de decisiones. permite enfatizar la dimensión creativa/constructiva de la preparación y desarrollo del proceso, el rol del docente como facilitador de entornos, la explicitación y discusión en torno a esos elementos, etc.

- Modelo situacional. Se pone en valor la importancia del contexto en la medida que condiciona estas decisiones en torno a la selección, secuenciación, organización, aplicación y análisis de contenidos, estrategias y tecnologías.

- Innovación Tic. El modelo TPACK puede contribuir a reorientar, centrar y filtrar los distintos usos educativos de las Tic. Desde el momento que se enfatiza la importancia de analizar el impacto del uso de las tecnologías, se reclama la necesidad de revisar críticamente las prácticas tecnológicas más innovadoras.

- La formación docente. a partir del análisis que propone el modelo se puede definir las competencias de los docentes para la adecuada integración de las Tic en su desempeño docente.

- Investigación educativa. El marco expuesto establece las bases para definir proyectos de investigación que permitan analizar las interacciones complejas entre contenido, pedagogía y tecnología. Esta iniciativa resultará imprescindible para superar los estudios basados en encuestas de opinión y avanzar hacia investigaciones más experimentales y centradas en la acción que permitan afianzar y mejorar los usos cotidianos de las Tic en el aula. (Gomez M. , 2018)

En este aspecto la utilización de las Tic en el proceso de aprendizaje requiere crear, mantener y restablecer un equilibrio dinámico entre los componentes del modelo, de acuerdo con Janssen y Lazonder citados por Salas (2018), el TPACK tiene un papel fundamental para mejorar las condiciones de enseñanza-aprendizaje ya que permite crear espacios atractivos e idóneos para la enseñanza, alcanzar los objetivos educativos de las asignaturas y facilitar el proceso de aprendizaje. Resulta valioso mencionar que esto permite la incorporación de 
cualquier tipo de tecnología en las actividades escolares. Por ejemplo, el uso de las herramientas web 2.0 como Wiki, Blogs, Facebook y videos son utilizadas durante la planeación, organización e implementación de las materias que se van a impartir.

\subsection{El modelo TPACK y las TAC}

Las Tecnologías de Aprendizaje y Conocimiento (TAC) se establecen como una reorientación de las Tic atendiendo más a la formación que a la información con el objetivo de lograr mejores aprendizajes incidiendo en los métodos y en cómo se utilizan las Tic y no únicamente en asegurar el dominio de una serie de herramientas informáticas (Vera Guadrón, y otros, 2020). En definitiva, se trata de conocer y explorar los diversos usos didácticos que las tecnologías tienen para el aprendizaje y la docencia.

Por otra parte se ha desarrollado una estrategia para aplicar el modelo TPACK a las TAC, se basa en un proceso de toma de decisiones de cinco momentos:

1. Seleccionar los objetivos de aprendizaje de la materia concreta que se va a impartir.

2. Determinar cómo van a ser las experiencias de aprendizaje.

3. Selección y secuenciación de las actividades.

4. Seleccionar las estrategias que se van a seguir para aplicar la evaluación formativa y sumativa.

5. Selección de las tecnologías más adecuadas para el desarrollo de las actividades propuestas.

En plena Sociedad de la Información y la Comunicación, el debate educativo se desplaza de la simple dotación a los docentes de conocimientos técnicos puramente informáticos a proporcionar una formación metodológica; es decir, tal contexto implica no sólo conocer las herramientas sino también saber seleccionar y utilizar adecuadamente las Tic para la adquisición de conocimientos y en función de las diferentes necesidades y perfiles de los estudiantes.

Autores Vallejo (2013), insisten en que el nuevo plan sobre el uso de las Tic pasa de ser un aprendizaje meramente manipulativo a un aprendizaje donde la tecnología aparece incluida en el proceso de enseñanza con metodologías precisas; es decir, un enfoque orientado al desarrollo de competencias clave. Es así como surgen los primeros planteamientos sobre normativa y orientación de las TAC en las aulas, así como nuevas propuestas metodológicas de inclusión de las Tic en los procesos de enseñanza y aprendizaje entre los que se encuentra el modelo TPACK; este modelo señala que los docentes deben poseer conocimientos pedagógicos y tecnológicos de la asignatura que imparte (Mishra, 2006, p. 1017)

El modelo TPACK aporta un punto de vista diferente respecto de la incorporación de las Tic en el aula, centrando la atención no en la capacitación exclusiva en competencias instrumentales sino en su interrelación con el componente didáctico. En este sentido, las competencias que debe poseer el docente son de tipo cognitivo, metodológico y actitudinal; su dominio y comprensión permitirán una utilización correcta de las tecnologías en su ejercico docente. (Molina, 2008, p. 318)

Autores como Escobar, Pujato y Medina citados por Ocaña (2013) mencionan que introducir de forma efectiva las TAC al proceso de enseñanza-aprendizaje supone una serie de cuestiones a tener en cuenta como son:

- La actualización continua de conocimientos.

- Las habilidades, procesos, y estrategias sobre los contenidos, tanto cognitivas como metacognitivas. 
- Una nueva conceptualización de la enseñanza como un proceso complejo en continuo cambio y que dura toda la vida.

- La generación de entornos virtuales de aprendizaje.

Por lo tanto Escobar (2007) afirman: "el docente debe dejar de ser un instructor que domina los conocimientos, para convertirse en un asesor, orientador, facilitador y mediador del proceso de enseñanza-aprendizaje" (p. 6). de igual forma el rol del estudiante también se ve modificado por las herramientas tecnológicas, pues esto implica una mayor motivación en el aprendizaje, así como un cambio de actitudes que enriquecen y contribuyen a ganar confianza en uno mismo (Pedró, 2011). Por tanto, el rol del estudiante ya no es un mero reproductor de contenidos memorísticos sino que debe llegar a ser un usuario inteligente y crítico de la información para lo que precisa aprender a buscar, obtener, procesar y comunicar información y convertirla en conocimiento.

La introducción de las TAC en la educación supone una serie de ventajas y desventajas que no se pueden obviar. A este respecto, Marquès Graells (2011), señala las siguientes:

Ventajas.

- Respecto al estudiante: Estas permiten el desarrollo de la iniciativa del mismo, aprendiendo de sus propios errores, de la relación con el docente a través de un aprendizaje cooperativo. Así mismo las TAC no sólo ayudan de forma intelectual, sino también visual, ya que facilitan el aprendizaje de conocimientos a estudiantes con necesidades educativas especiales.

- Respecto al docente: son una fuente ilimitada de recursos educativos para la docencia y atención del estudiante, de igual forma facilitan la investigación en el aula.

\section{Desventajas.}

- La forma incorrecta pueden llevar a los estudiantes, e incluso a docentes, a sufrir una gran distracción y dispersión de la tarea principal.

- Si no se extraen informaciones útiles o válidas, puede suponer una visión parcial de la realidad, que no llega a entrar en la estructura de conocimientos del estudiante, siendo una pérdida de tiempo.

- La adicción de recursos tecnológicos pueden provocar aislamiento social en cuanto al resto de compañeros.

Pese a estas ventajas y desventajas, la inclusión de la tecnología en la educación es un recurso pedagógico que facilita la adquisición del aprendizaje y son un elemento motivador dentro del aula ya que han impactado positivamente los resultados académicos. Motivo por el cual, el papel de los docentes y las autoridades de las Instituciones Educativas es clave en esta integración y construcción de infraestructura tecnológica cuyo propósito es la de integrar la nueva cultura denominada alfabetización digital.

\subsection{Formación docente en las tecnologías de aprendizaje}

Por sí mismas, las Tic no dan lugar a una optimización de la enseñanza-aprendizaje; muchas escuelas e institutos han dejado de apoyar o de recibir ayudas estatales para apoyar esta integración. Por otra parte, la mejor manera para aprender cómo integrar efectivamente la tecnología en el aula es a través del aprendizaje situado. Esto sucede cuando aprendemos nuevos conocimientos y habilidades en un entorno integrado dentro del contexto de enseñanza por ejemplo el desarrollo profesional De Diseño, donde los docentes rediseñan sus cursos y lecciones para dar lugar a otras diferentes con la tecnología integrada, pero sobre la base previa del contexto real y de modo participativo con otros colegas (Merhbi, 2020). 
El docente debe saber manejar su creatividad de manera que esta interacción suponga una mejora real de la calidad de la enseñanza, integrando los conocimientos pedagógicos, disciplinares y tecnológicos. Esto significa que son ellos los que deben dar una solución creativa a la hora de enseñar dentro y fuera del aula, integrando un conjunto de conocimientos que van de la mano con el diseño instruccional.

Las Tic generan un impacto significativo en la enseñanza del docente y en el aprendizaje del estudiante, son como un medio que apoyan la instrucción; al respecto Mazzotti Díez (2016), mencionan que el uso de la tecnología permite cambios como: (a) una mejora en las actividades áulicas; (b) la habilitación de diferente patrones de comunicación; (c) la búsqueda de oportunidades del docente para diseñar actividades que desarrollen la creatividad, las habilidades de pensamiento, las competencias lingüísticas y otras destrezas intelectuales, socioemocionales y personales.

El docente necesita apropiarse de las tecnologías y para ello tiene que adaptar los diseños y los procesos de sus planificaciones a nuevas formas de enseñar, en consonancia con las teorías de aprendizaje que muestran la importancia de centrar las actividades en el estudiante. Por su parte, la investigación llevada a cabo por Brualdo y Sabogal (2015) evidencia tres concepciones diferentes acerca del uso de las Tic: (a) una perspectiva puramente instrumental; (b) como herramientas que potencian los aprendizajes; (c) concebidas como diseño, potenciando las intencionalidades pedagógicas (Mazzotti Díez, 2016).

Varios estudios (Afshari, Abu Bakar, Su Luan, Abu Samah, \& Say Fooi, 2009; Schiller, 2003; Rogers, 1995) plantean como hipótesis de investigación que la adopción de una innovación por parte de un docente está condicionada por factores como el nivel educativo, la edad, el género, la experiencia profesional, las competencias y habilidades en el uso de las tecnologías, el conocimientos de las potenciales didácticas de las Tic, las actitudes frente a la tecnología, las creencias personales sobre el potencial pedagógico de las mismas y las barreras en los procesos de innovación. (Mazzotti Díez, 2016, p. 56)

Las características personales pueden influir en la forma en que los docentes utilizan las aplicaciones tecnológicas en las aulas; Mazzotti Díez (2016) afirma: "Un docente con un pensamiento creativo tiende a utilizar las TIC para la construcción del conocimiento" (p. 57); es una persona que busca actualizarse permanentemente, que estudia, que intercambia materiales y reflexiones con sus colegas por ende es más propenso a usar la tecnología de forma integradora e innovadora. Rogers (1995) clasifica a los docentes en cinco categorías:

Tabla 1

Tipos de Docentes en la Innovación Educativa

\begin{tabular}{|c|c|c|}
\hline \multirow{3}{*}{ 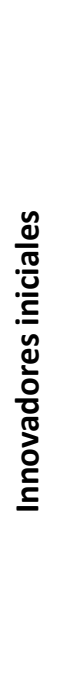 } & Adoptantes tempranos & $\begin{array}{l}\text { Conforman un grupo de docentes que se encuentra en la búsqueda de formas de } \\
\text { inclusión de la innovación así mismo muestra empatía, poco dogmatismo, gran } \\
\text { capacidad para hacer frente a abstracciones con racionalidad, mantienen una } \\
\text { actitud entusiasta hacia el cambio, con aptitud para enfrentar la incertidumbre y el } \\
\text { riesgo, poco fatalismo y aspiraciones elevadas. }\end{array}$ \\
\hline & Adoptantes avanzados & $\begin{array}{l}\text { Este grupo son docentes que ya han incorporado la innovación; se caracterizan por } \\
\text { ser muy realistas sobre las posibilidades que ofrecen las Tic, firmes en sus juicios, } \\
\text { con disposición a superar las dificultades y problemas, críticos y cautos con las } \\
\text { modas. }\end{array}$ \\
\hline & Reticentes al cambio & $\begin{array}{l}\text { Están poco dispuestos a correr riesgos innecesarios y en sus decisiones ponderan el } \\
\text { criterio adquirido a través de la experiencia profesional; se sienten inseguros en el } \\
\text { aula informática y temen perder el control de la situación al no ser ellos el centro } \\
\text { del modelo educativo frente a los estudiantes que sospechan que maneja los } \\
\text { ordenadores con mucha más soltura que ellos. }\end{array}$ \\
\hline
\end{tabular}


Nota: Recuperado de "Los ITICnerarios docentes. Enseñantes recorriendo territorios tecnológicos", Mazzotti D. 2016, Universidad ORT Uruguay, p. 56-57.

En cualquiera de los casos, se debe tener presente que todo ello implica un esfuerzo de formación y de tiempo para los docentes y que estos no deben ser infravalorado por la institución. En el proceso de aprendizaje de las nuevas tecnologías, son muchas las cosas que cambian, sin embargo, conviene tener presente que en cualquier sistema educativo de calidad la pieza clave que determina el éxito del programa continúa siendo el docente. En el ámbito pedagógico deben tener capacidad para enseñar a los estudiantes a aprender con las herramientas proporcionadas por las nuevas tecnologías y a conocer cómo utilizarlas para sacarles el mejor provecho.

\subsection{Objetivo}

Determinar la aplicabilidad docente del modelo TPACK el cual promueve el uso de herramientas tecnológicas considerando los conocimientos pedagógicos y disciplinares (Cabero, 2015). Es dentro de este marco en el que se ubica la presente investigación, desarrollada en las instituciones educativas de tipo fiscal, fiscomisional y municipal del Distrito 11D01 de la ciudad de Loja, donde se desea analizar el impacto en el rendimiento académico de primer quimestre del año lectivo 2019-2020 de los estudiantes de Educación General Básica Elemental.

\section{Metodología}

\subsection{Metodo}

El estudio de esta investigación científica es de tipo descriptivo-correlacional se realizó en las Instituciones Educativas de Educación General Básica Elemental de tipo fiscal, fiscomisional y municipal del Distrito 11D01 de la ciudad de Loja. Cabe mencionar que el estudio de este tipo según Fidias G. (2012) afirma: "consiste en la caracterización de un hecho, fenómeno, individuo o grupo con el fin de establecer su estructura; es un tipo de estudio que tiene como propósito evaluar la relación que existe entre dos o más conceptos, en un contexto en particular" (p. 43). Partiendo así del método deductivo que va de lo general a lo particular y considerando los juicios que se formulan a partir de determinadas proposiciones, planteados en base a las variables del tema propuesto, se determinó la aplicabilidad docente del modelo TPACK en el transcurso del primer quimestre del año lectivo 2019-2020.

\subsection{Descripción del contexto}

La muestra utilizada en esta investigación consiste en 81 docentes de Educación General Básica Elemental de la Zona 7, Distrito 11D01 de Ecuador, pertenecientes a 3 Instituciones Educativas de tipo fiscal, fiscomisional y municipal, en el transcurso del primer quimestre del año lectivo 2019-2020 de la ciudad de Loja, país Ecuador.

\subsection{Instrumento}

La presente investigación utiliza un cuestionario ad hoc, para docentes denominado "Aplicabilidad docente del modelo TPACK para EGBE", que contempla 5 dimensiones, para los tres tipos de Institución Educativa, fiscal, fiscomisional y municipal, como se detallan a continuación:

- Conocimiento Pedagógico del Contenido (PCK): (Pedagogical Content Knowledge, PCK), en referencia a la idea de Shulman, se plantea como la transformación de la materia para la enseñanza, la cual ocurre cuando el docente adapta el contenido a los conocimientos previos de los estudiantes (Koehler y Mishra, 2009, p. 60). 
- Conocimiento Didáctico del Contenido (DCK): (Didactic Content Knowledge, DCK), hace mención a transformar la información de una materia determinada para aplicarla a la enseñanza de una manera atractiva e innovadora (Shulman, 1986, p. 5).

- Conocimiento Tecnológico del Contenido (TCK): (Technological Content Knowledge, TCK) plantea que los docentes necesitan dominar el contenido de la materia que enseñan y tener un profundo conocimiento en la manera en que el objeto pueden cambiarse mediante la aplicación de tecnologías particulares (Pamuk, 2012). Los docentes necesitan saber qué tecnologías específicas son las más adecuadas para abordar el aprendizaje de su dominio y cómo el contenido cambia la tecnología, o viceversa.

- Conocimiento Tecno-Pedagógico del Contenido (TPACK): está comprometido con una enseñanza significativa, que requiere la comprensión en la representación de conceptos en el uso de tecnologías, apoyadas con técnicas pedagógicas; de modo que, se pueda enseñar el contenido y que esté a su vez pueda utilizarse para construir sobre el conocimiento existente, nuevas epistemologías (Koehler y Mishra, 2009, p. 67)

Cabe mencionar que la aplicación de este Instrumento de Medición (encuesta) fue elaborado en Google Forms y aplicado en línea como complemento para determina la aplicabilidad del modelo TPACK en el ejercicio docente.

\subsection{Procedimiento}

El procedimiento de esta investigación inició con la selección de la metodología científica de tipo descriptivocorrelacional; a continuación se elaboró el cuestionario ad hoc para docentes denominado "Aplicabilidad docente del modelo TPACK para EGBE"; luego se realizó la elección de las Instituciones Educativas (IE) de tipo fiscal, fiscomisional y municipal de la Zona 7, Distrito 11D01 de Ecuador, para realizar las respectivas solicitudes por escrito a las autoridades de las 3 instituciones solicitando el permiso correspondiente para la aplicación de una encuesta en línea a los docentes de EGBE por Google Forms; seguidamente se permitió la recolección y procesamiento de los datos, para posteriormente realizar el análisis y las conclusiones finales.

\section{Resultados}

La información recopilada con respecto a la muestra de los docentes de EGBE pertenecientes a 3 Instituciones Educativas de tipo fiscal, fiscomisional y municipal, se presentan a continuación en gráficas por medio de un análisis descriptivo-correlacional:

Figura 1

Conocimiento Pedagógico del Contenido (PCK)

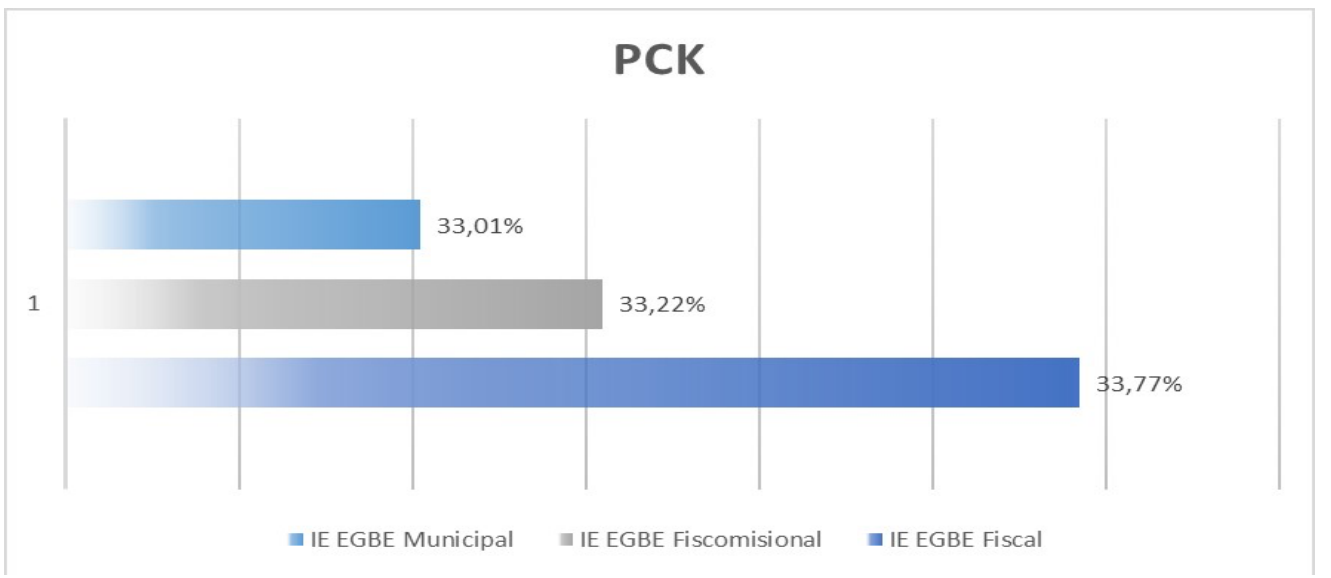

Nota: Encuesta aplicada a docentes de las IE EGBE: Fiscal, Fiscomisional y Municipal. 
Según los datos (Figura 1) sobre el Conocimiento Pedagógico del Contenido (PCK), el 33,77\% de los docentes en IE EGBE Fiscales manifestaron haber adaptado el contenido de la materia a los conocimientos previos de los estudiantes, mientras que el $33,22 \%$ en IE Fiscomisionales y un $33,01 \%$ en las IE Municipales.

En efecto se observa que la totalidad de los docentes en el transcurso del primer quimestre adaptaron los materiales didácticos disponibles para el desarrollo de clase, tomando en cuenta el Plan de Unidad Didáctica (PDU) y los conocimientos previos del estudiante.

Contrastando con Koehler y Mishra (2009) difiere sobre el conocimiento pedagógico del contenido (PCK) se plantea como la transformación de la materia para la enseñanza, la cual ocurre cuando el docente adapta el contenido a los conocimientos previos de los estudiantes.

Figura 2

Conocimiento Didáctico del Contenido (DCK)

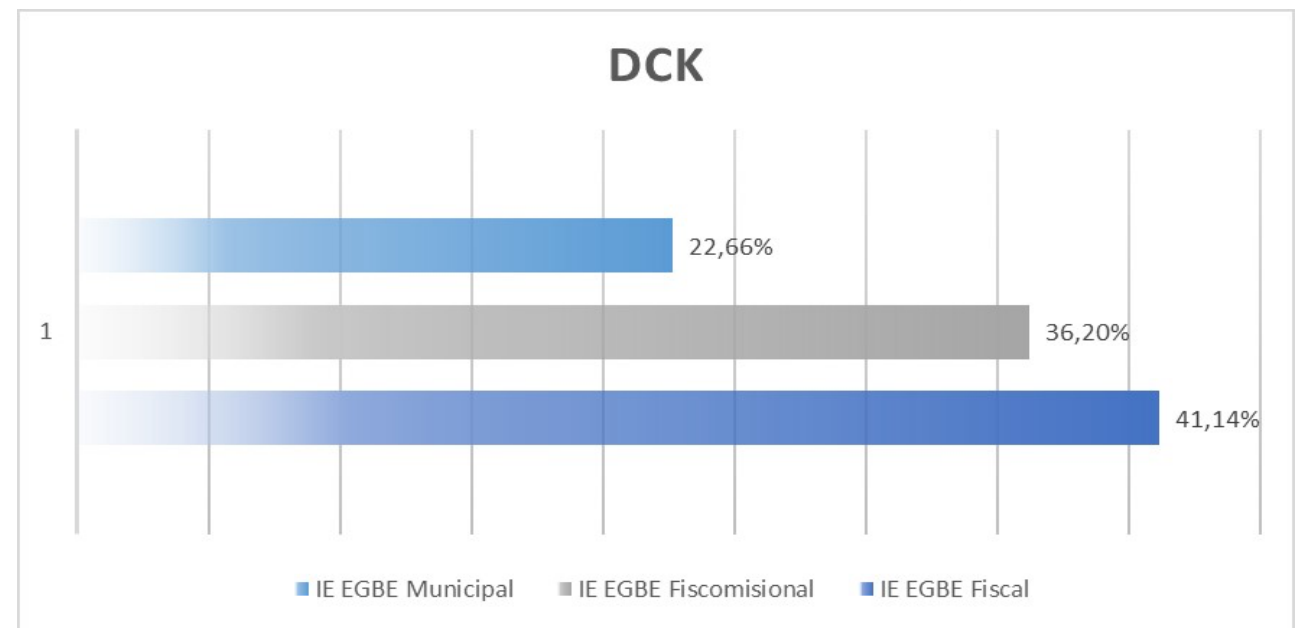

Nota: Encuesta aplicada a docentes de las IE EGBE: Fiscal, Fiscomisional y Municipal.

Según los datos (Figura 2) sobre el Conocimiento Didáctico del Contenido (DCK), el 41,14\% de los docentes en IE EGBE Fiscales manifestaron haber transformado la información de la materia de una manera más atractiva e innovadora utilizando las Tic, mientras que el $36,20 \%$ en IE Fiscomisionales y un 22,66\% en las IE Municipales.

En consecuencia se observa que los docentes encuestados en las IE EGBE municipales son los que menos innovaron el desarrollo de la clase con la ayuda de las Tic; mientras que entre las IE Fiscales y Fiscomisionales se observa solo un 4,94\% de contraste al momento de impartir los contenidos durante el primer quimestre.

Corroborando con Shulman citado por Pinto Sosa y González Astudillo (2008) el CDK no se limita a estudiar cómo se enseña para obtener conocimiento de la didáctica general, sino que busca que el docente comprenda lo que se ha de aprender y cómo se debe enseñar el contenido a partir de la comprensión de cómo el estudiante aprende y comprende, resuelve problemas y desarrolla su pensamiento crítico. 
Figura 3

Conocimiento Tecnológico del Contenido (TCK)

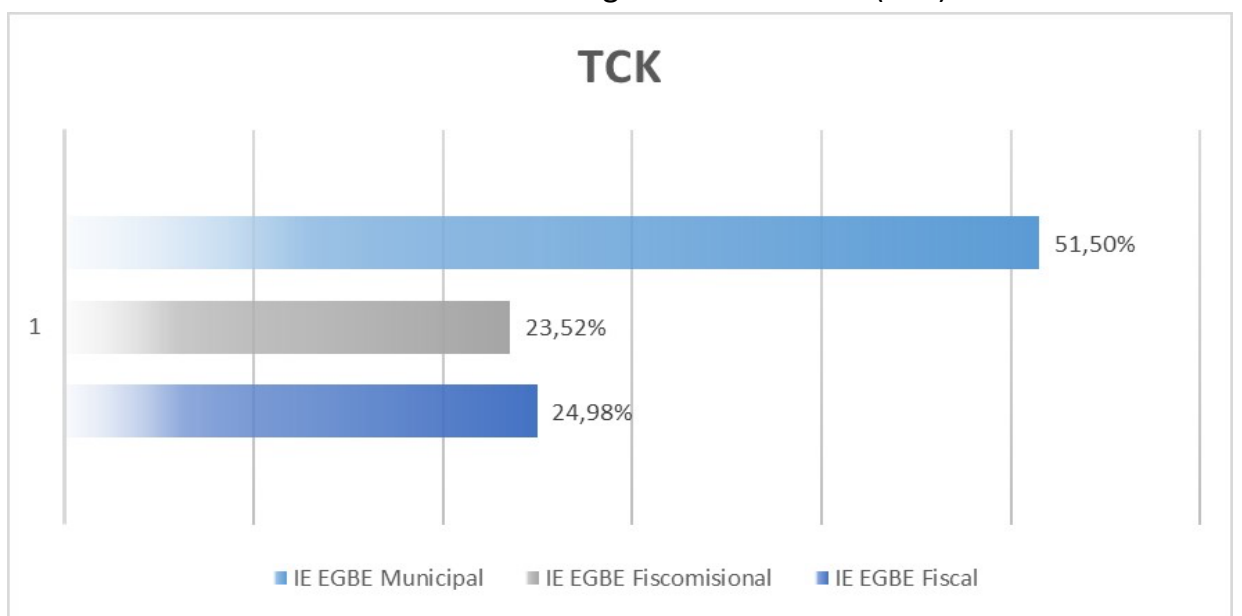

Nota: Encuesta aplicada a docentes de las IE EGBE: Fiscal, Fiscomisional y Municipal

Según los datos (Figura 3) sobre el Conocimiento Tecnológico del Contenido (TCK), el 51.50\% de los docentes en IE EGBE Municipales mostraron haber utilizado las Tic para el desarrollo de la clase en la presentación del contenido, así como conocer qué tecnologías específicas son más adecuadas para abordar la enseñanza y el aprendizaje, mientras que el $24,98 \%$ en IE Fiscales y un $23,52 \%$ en las IE Fiscomisionales.

En consecuencia se observa que los docentes encuestados en las IE EGBE Municipales son los que mostraron tener conocimiento sobre qué tecnologías son las más adecuadas para enseñar una materia concreta; mientras que en las IE fiscomisionales y municipales se observa un conocimiento menor sobre cómo diferenciar entre qué herramienta es mejor para el aprendizaje al momento de impartir los contenidos.

Contrastando con Tourón (2016) refiere a que los docentes necesitan entender qué tecnologías específicas son las más adecuadas para abordar el aprendizaje objeto en sus dominios y cómo el contenido dicta o quizás incluso cambia la tecnología, o viceversa.

Figura 4

Conocimiento Tecno-Pedagógico del Contenido (TPACK)

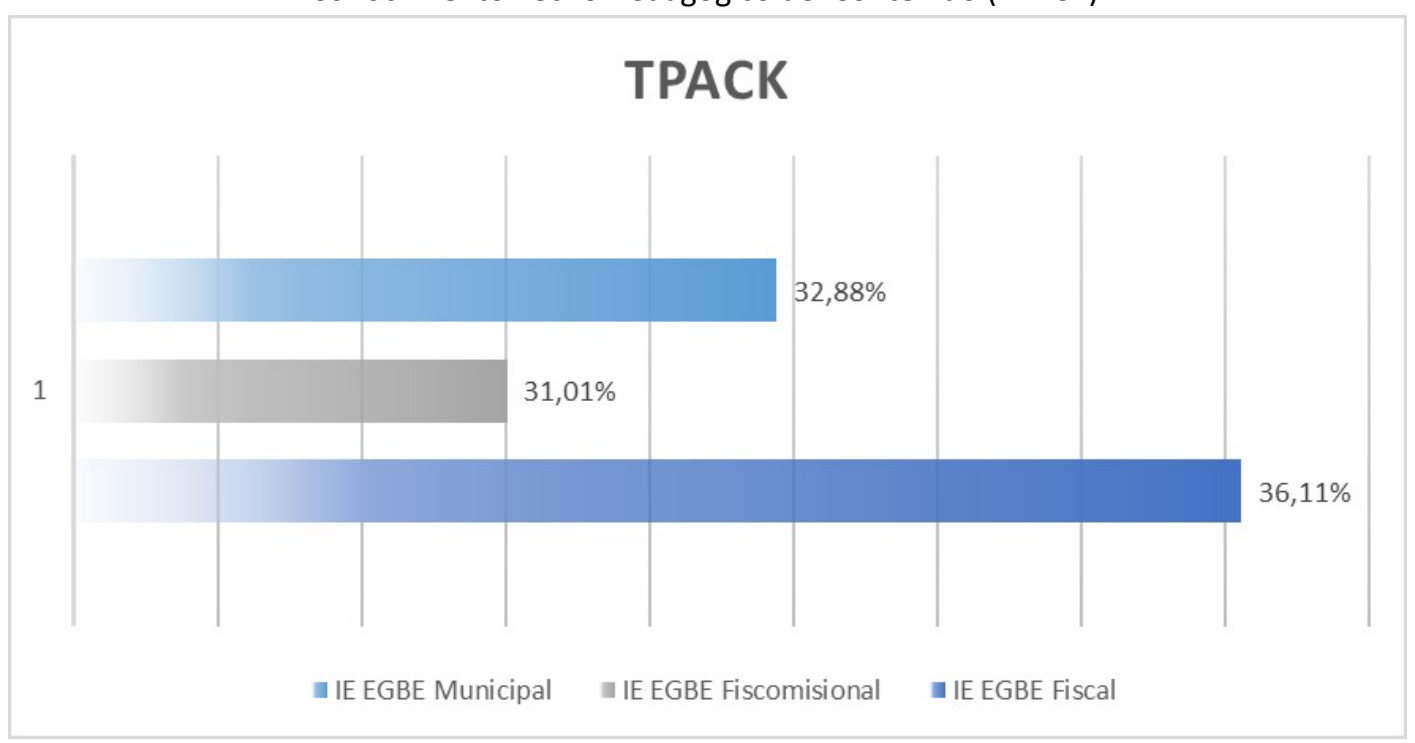

Nota: Encuesta aplicada a docentes de las IE EGBE: Fiscal, Fiscomisional y Municipal 
Según los datos (Figura 4) sobre el Conocimiento Tecno-Pedagógico del Contenido (TPACK), el 36,11\% de los docentes en IE EGBE Fiscales manifestaron que el modelo TPACK es la base de la enseñanza efectiva con la tecnología ya que esta puede ayudar a corregir algunos de los problemas que afrontan los estudiantes al momento de aprender con las Tic; mientras que solo el $32,88 \%$ en IE Municipales y un $31,01 \%$ en las IE Fiscomisionales.

En efecto se observa que los docentes de las IE Fiscales en el transcurso del primer quimestre aplicaron el modelo TPACK en el desarrollo de sus clases dando lugar a nuevas epistemologías del conocimiento y fortaleciendo las ya existentes sin dejar a un lado el contexto en el que se desarrolla el estudiante.

Contrastando con Vallejo (2013) menciona que el modelo TPACK es la integración de todos los componentes técnicos, pedagógicos y contenidos. Supone integrar lo que el docente sabe sobre la materia que desea impartir, los métodos didácticos más adecuados a la situación concreta de los estudiantes, sabe cómo integrar la tecnología para enseñar mejor un contenido concreto.

\section{Conclusiones}

EI TPACK constituye un marco teórico interesante para una integración eficaz de las tecnologías en la enseñanza. Reconoce la importancia de los tres componentes fundamentales: contenido, pedagogía y tecnología, centrando el foco en las múltiples interacciones que existen entre ellos. Los resultados obtenidos a través de las encuestas prescriben que el modelo apoya a los docentes de las IE Fiscales durante la exploración y selección de las herramientas digitales idóneas para el desarrollo de las competencias en los estudiantes. Por ende, se evidenció un crecimiento en el rendimiento académico en el transcurso del primer quimestre del año lectivo 2019-2020 en relación con años anteriores.

La parte esencial del estudio realizado es que a partir de la adquisición de los componentes básicos del modelo se observa la predisposición positiva al uso de las Tic a partir de un diseño pedagógico previo y no como simple herramienta sustituta de la pizarra o el libro tradicional. Además se ha observado que los ítems presentados en las encuestas sobre la Aplicabilidad docente del modelo TPACK para EGBE muestran una frecuencia de respuestas "de acuerdo" o "totalmente de acuerdo" lo que indica que el modelo desarrollado puede ser una forma nueva y funcional de incorporación de las Tic en las aulas de Grado y, en consecuencia, de Elemental.

En cuanto al Conocimiento Pedagógico del Contenido (TCK) se puede afirmar que los docentes de las Instituciones Educativas de EGBE disponen de una percepción muy buena en la adaptación del contenido con los materiales didácticos, tomando en consideración el modelo constructivista. Referente al análisis del Conocimiento Didáctico del Contenido (DCK) se evidencio que hay un alto porcentaje de aplicabilidad de este en las IE EGBE fiscales y fiscomisionales frente a las municipales, esto se debe a que los docentes carecen de algunos conocimientos de contenido disciplinar, por ende como hemos señalado en este artículo, el no innovar la forma de presentar la materia al momento de desarrollar la clase, limita la capacidad de los estudiantes de aprender a aprender, y lo que es también relevante en la inclusión de la tecnología para la educación.

Frente a ello, los docentes de las IE EGBE municipales son conscientes de la necesidad del elemento tecnológico de contenido para la construcción del conocimiento; asi mismo de acuerdo a las respuestas en la encuesta vinculadas al (TCK), mostraron dominio al momento de saber qué recursos tecnológicos son los más adecuados para enseñar las materias. Finalmente, en cuanto a la aplicabilidad del modelo TPACK los docente de las Instituciones Educativas fiscales manifestaron que es la base de la enseñanza, ya que esta puede ayudar a corregir algunos de los problemas que afrontan los estudiantes al momento de aprender con las Tic, sin dejar a un lado las otras instituciones que también se vieron interesados en la aplicabilidad de este modelo a sus prácticas docentes. 
Todo esto nos da la pauta para permitirnos avanzar en la inclusión de los contenidos con la tecnología junto a la pedagogía como una sola, en la que se desean transmitir los saberes en pro de la formación del estudiante; es aquí donde al utilizar las Tic para mejorar los procesos de enseñanza-aprendizaje empezamos a hablar de las TAC, convirtiéndose ambas en el aliado perfecto para el desarrollo de un método global de conocimiento que tenga en cuenta las destrezas de pensamiento histórico vinculadas al modelo TPACK.

\section{Referencias bibliográficas}

Adell, J. (2012). Diseño de Actividades según el TPACK. Fragmento de la charla "Diseño de actividades didácticas con TIC" [Archivo de video]. Recuperado de https://www.youtube.com/watch?v=5mi2D7WTMXI

Afshari, M., Abu Bakar, K., Su Luan, W., Abu Samah, B., \& Say Fooi, F. (2009). Factores que afectan el uso de tecnología de información y comunicación por parte de los docentes. En Revista Internacional de Instrucción, 2(1), 77-104.

Barajas Alcalá , L., y Cuevas Salazar , O. (2017). Adaptación del modelo tpack para la formación del docente universitario. San Luis Potosí.

Brualdo, M., y Sabogal, M. (2015). Trayectos de uso de las TIC: Caso de la Universidad Javeriana. Revista Internacional de Investigación en Educación, 135-148.

Cabero, J. M. (2015). Validación de la aplicación del modelo TPACK para la formación del profesorado en TIC. Revista de innovación educativa, 14(13). .

Castillejos, B., Torres Gastelú, C. A., y Lagunes Dominguez, A. (2016). Capitulo III El Entorno Personal de Aprendizaje y el modelo TPACK en la mejora educativa. Mexico: Instituto Tecnológico de Sonora.

Coll, C. (2004). Psicología de la educación y prácticas educativas mediadas por las tecnologías de la información y la comunicación. Una mirada constructivista. Sinéctica Revista electrónica de Educación, 25. Recuperado de https://sinectica.iteso.mx/index.php/SINECTICA/article/view/277

Dussel, I. (2010). VI Foro Latinoamericano de Educación; Educación y nuevas tecnologías: los desafíos pedagógicos ante el mundo digital. Buenos aires: Santillana. Recuperado de http://www.virtualeduca.org/ifd/pdf/ines-dussel.pdf

Escobar, S. M., Pujato, L., C, C., y Medina Bravo, C. J. (2007). Las TIC en la Educación: panorama internacional y situación española. XXII Semana Monográfica de la Educación. España: Santillana.

Fidias, G. (2012). Metodologías activas para la formación de competencias. Education siglo XXI, 24.

Gomez, M. (26 de Enero de 2018). E-learning Masters. Obtenido de ¿Sabes qué es el TPACK y cómo implementarlo en tus cursos? Recuperado de http://elearningmasters.galileo.edu/2018/01/26/sabes-quees-el-tpack-y-como-implementarlo-en-tus-cursos/

Gomez, M. M. (26 de Enero de 2018). E-learning Masters. Obtenido de ¿Sabes qué es el TPACK y cómo implementarlo en tus cursos? Recuperado de http://elearningmasters.galileo.edu/2018/01/26/sabes-quees-el-tpack-y-como-implementarlo-en-tus-cursos/

González, N. V. (2017). Influencia del contexto en el desarrollo del conocimientotecnológico pedagógico del contenido (TPACK) de un profesor universitario. Argentina.

Harris, J. B. (15 de Julio de 2012). Wiki de tipos de actividades de aprendizaje. Recuperado de http://activitytypes.wmwikis.net/HOME

Janssen, N. y. (2015). Implementación de tecnologías innovadoras a través de planes de lecciones: ¿Qué tipo de apoyo prefieren los maestros? . Revista de Ciencia, Educación y Tecnología, 24(6). 
Koehler, M., y Mishra, P. (2009). ¿Qué es el conocimiento tecnológico de contenido pedagógico (TPACK)? Problemas contemporáneos en tecnología y formación docente. 9(1), 60-70.

Marquès Graells, P. (07 de Agosto de 2011). Impacto de las TIC en educación: funciones y limitaciones. Recuperado de http://peremarques.pangea.org/siyedu.htm

Mazzotti Díez, W. (2016). Los iTICnerarios docentes Enseñantes recorriendo territorios tecnológicos. Uruguay.

Merhbi, A. (09 de Abril de 2020). Proyecto escuela 2.0 una nueva educación para todos. Obtenido de Obtenido de TPACK: ¿Qué necesitan saber los/as docentes para integrar efectivamente las TIC? Recuperado de: http://www.escuela20.com/tpack-tic-tpack/articulos-y-actualidad/tpack-que-necesitan-saber-losasdocentes-para-integrar-efectivamente-las-tic_2806_42_4299_0_1_in.html

Mishra, P., \& Koehler, M. J. (2006). Contenido pedagógico tecnológico: Un nuevo marco para el conocimiento del maestro, 108(6).

Molina, G. M. (2008). Competencias del profesorado para el uso de las tecnologías de la información y la comunicación en la enseñanza, en el marco del Espacio Europeo de Educación Superior. Dialnet, 317-326.

Ocaña, M. C. (2013). La Integración de las TAC en Educación. Valencia: UNIR.

Pamuk, S. (2012). Comprender el uso de la tecnología de los maestros en servicio a través del marco TPACK. 28(5), 425-439.

Pedró, F. (2011). Tecnología y Escuela: lo que funciona y por qué. XXVI Semana Monográfica de la Educación. La Educación en la Sociedad Digital. España: Santillana.

Pinto Sosa, J. E., y González Astudillo, M. T. (2008). El conocimiento didáctico del contenido en el profesor de matemáticas: ¿una cuestión ignorada? Redalyc, 20(3), 83-100. Recuperado de https://www.redalyc.org/pdf/405/40512064005.pdf

Rogers, E. (1995). Difusión de innovaciones. Revista Internacional de Educación, 67.

Salas, R. A. (2018). Uso del modelo TPACK como herramienta de innovación para el proceso de enseñanzaaprendizaje en matemáticas. SCIELO, 24.

Schiller, J. (2003). Trabajando con las percepciones TIC de los directores australianos. Journal of Educational Administration, 41(2), 171-185.

Shulman, L. S. (1986). Los que entienden: el crecimiento del conocimiento en la enseñanza. Investigador educativo, 15(2), 4-14.

Tourón, J. (18 de Noviembre de 2016). TPACK: Un modelo para los profesores de hoy. Recuperado de https://ined21.com/tpack/

Vallejo, C. (04 de Abril de 2013). Observatorio Tecnológico. Introducción de las tecnologías en la educación TPACK. Recuperado de http://recursostic.educacion.es/observatorio/web/fr/cajon-de-sastre/38-cajon-desastre/1092-monografico-introduccion-de-las-tecnologias-en-la-educacion?start=1

Vera Guadrón, L. J., Gómez, S., Lordes, M., Acosta B, Coromoto, Y., . . Lorehny. (25 de Junio de 2020). La docencia en el marco de la responsabilidad social universitaria. Opción, 28 (68). Recuperado de https://www.redalyc.org/articulo.oa?id=310/31025437013

Esta obra está bajo una Licencia Creative Commons Attribución-NoCommercial 4.0 International

(cc) $\mathrm{BY}-\mathrm{NC}$ 